\title{
Role of global femoral cartilage in assessing severity of primary knee osteoarthritis
}

\author{
Naylan Fayez Wahba Khalil ${ }^{1 *}$, Sherine El-sherif ${ }^{1}$, Mowaffak Moustafa Abd El Hamid ${ }^{1}$, Rehab Elnemr ${ }^{1}$ and \\ Raghda Saad Zaghloul Taleb²
}

\begin{abstract}
Background/objective: Osteoarthritis is a degenerative joint disease marked by structural changes in the joint. Radiological evaluation can be used to assess structural changes. Pain, inflammation, and stiffness are common clinical symptoms, leading to limitations in daily activities. Ultrasound, unlike traditional radiography, allows for a direct examination of changes in soft tissues. In addition, it is sensitive in detecting osteophytes as well as identifying early OA changes in femoral cartilage associated with clinical manifestations and function.

Results: A cross- sectional study of 40 patients with primary KOA diagnosed according to the American College of Rheumatology (ACR) criteria. After radiographic evaluation using Kellgren-Lawrence (K-L) scale and US examination assessing global femoral hyaline cartilage (GFC), osteophytes, meniscal extrusion, effusion, and Baker's cyst of the most symptomatic knee, there was significant correlation between (K-L) grading and (GFC) ultrasonographic grading $(p=<0.001)$. After assessment of pain and functional disability using Western Ontario and McMaster Universities Osteoarthritis Index (WOMAC) scale, there was significant correlation between $\mathrm{KL}$ and GFC grading with age $(p=<0.001$ for both), disease duration ( $p=<0.001$ for both) as well as WOMAC total scores ( $p=<0.001$ for both). GFC grading was the only independent predictor relative to other ultrasonographic variables for WOMAC total score $(p=<0.001)$.

Conclusions: US is a valid tool to evaluate knee joint space and is well correlated with radiographic images. KOA severity assessed by KL grading and GFC ultrasonographic grading showed good correlation with age, duration of the disease, pain intensity, and functional disability.
\end{abstract}

Keywords: Knee, Osteoarthritis, Ultrasound

\section{Background}

Knee osteoarthritis (KOA) is one of the most frequent degenerative joint disease in the elderly, causing pain and limitation of joint function [1]. Previous research has shown that only about half of patients with knee pain suspecting KOA will have radiographic changes [2]; moreover, only about half of patients with radiographic KOA might experience pain [3]. There is moderate relationship between the radiographic degree of $\mathrm{KOA}$ and the level of

\footnotetext{
*Correspondence: naylanfayez55@gmail.com

1 Physical Medicine, Rheumatology and Rehabilitation Department,

Faculty of Medicine, Alexandria University, Alexandria, Alexandria

Governorate 21131, Egypt

Full list of author information is available at the end of the article
}

pain $[4,5]$. Conventional radiography $(\mathrm{CR})$ was the first imaging modality used to diagnose $\mathrm{KOA}$, which assesses the bone structure and severity of the disease. The evaluation of both osteophytes and joint space narrowing is the mainstay of CR assessment of KOA [6], although has a limited ability to image soft tissues and gives indirect evidence of cartilage destruction $[7,8]$.

Ultrasonography (US) has recently improved KOA diagnosis and management [9]. It is a practical, reliable, noninvasive, cheap, readily available, valid tool for assessing KOA and does not need the use of ionizing radiation [10]. Current US technology has a number of advantages, including the capacity to identify osteophytes, articular cartilage degeneration, meniscal and ligament tears, bursitis, effusion, and interventional operations [11]. Several 
researches have reported significant correlations between KOA characteristics as detected by US and CR, especially for osteophytes where ultrasound is more sensitive than radiography [12].

The aim of this study was to evaluate the association between clinical examination and functional disability with ultrasonographic and radiographic findings in patients suffering from primary (KOA).

\section{Methods}

This cross-sectional study was performed at the Physical Medicine, Rheumatology and Rehabilitation outpatient clinics in the Main University Hospital. Forty patients fulfilling the ACR criteria for KOA [13] were enrolled in the present study. Patients with secondary KOA, history of previous knee surgery, and trauma were all ruled out.

Patient evaluation was performed following the International Classification of Functioning, Disability and Health (ICF) [14]; all patients were subjected to detailed local knee assessment, and WOMAC scores were used to assess pain and functional disability. Plain anteroposterior weight-bearing radiographs of both knees and musculoskeletal US of the more symptomatic knee were performed.

\section{A. Impairment was assessed based on:}

\section{Clinical examination of the knee joint}

Detailed clinical examination of the knee was done with stress on alignment, swelling (bony, soft tissue as Baker's cyst), tenderness (tested using Doyle Index) [15, 16], effusion, crepitus, and WOMAC OA index $[17,18]$ (was used to assess either knee pain by WOMAC pain subscale or knee stiffness by WOMAC stiffness subscale).

\section{Radiological assessment of affected knee joint}

Plain X-Ray of both knees in standing anteroposterior was done, and the radiological KOA severity was assessed by using the Kellgren-Lawrence (KL) global scale [19].

\section{Sonographic assessment}

Ultrasound of the knee was done to assess KOA severity and associated soft tissue abnormality [5]. The examination was performed by two rheumatologist ultrasonographers (ES S) and (AF Y) with high experience in musculoskeletal US using a $3-16-\mathrm{MHz}$ linear array transducer (Samsung HS50, Korea). All settings were kept constant for each patient throughout the data collection except focus and image depth.
The subject was positioned supine with full flexion of the knee. Medial, sulcus, and lateral femoral articular cartilages were scanned in a transversal plane by proximal-distal probe sweeping over the anterior-central knee area to assess the cartilage of the femoral sulcus and the medial and lateral femoral condyles. The entire cartilage area reachable by US through the acoustic window was scanned. Cartilage was considered degenerated when loss of surface sharpness, increased inner echogenicity, local thinning, and/or total loss of cartilage thickness were observed. In a transverse plane and with the knees in maximum flexion, the femoral hyaline cartilage was assessed and classified in 5 degrees: 0 , normal; 1 , loss of normal sharpness level interfaces or increased echogenicity cartilage; $2 \mathrm{~A}$, modification from degree 1 with decreasing the cartilage thickness $<50 \%$ of his size; $2 \mathrm{~B}$, decreasing the cartilage thickness $>50 \%$, but $<100 \%$; and, $3,100 \%$ local loss of cartilage thickness [20]. Then, the subject was asked to fully extend the knee, and medial and lateral femoral and tibial osteophytes as well as medial and lateral menisci were longitudinally imaged by anterior-posterior scanning from the medial and lateral side of the joint space and were subsequently evaluated for osteophyte presence and size using validated grading systems as follows: grade $0=$ absence of osteophyte, grade $1=$ minimal osteophyte, grade $2=$ medium-sized osteophyte, and grade $3=$ large osteophyte [21]. Meniscal extrusion was confirmed if it protruded $>3 \mathrm{~mm}$ perpendicularly from the joint line connecting the tibial and femoral bone ends [21].

\section{B. Activity and participation}

WOMAC score with the physical function subscale was used to assess the functional disability of the patients [18].

\section{Statistical analysis}

All statistical analyses were performed with IBM SPSS software package version 20.0. (Armonk, NY: IBM Corp) [22]. Continuous data were expressed as mean $\pm S D$, whereas categorical data were expressed in number and percentage. The differences between two groups or more were determined using independent sample Student's $t$-test or one-way analysis of variance test, respectively, for variables with continuous data or the $\chi^{2}$ test for variables containing categorical data $\left(\chi^{2}\right)$ test $(P<0.05$ was considered statistically significant). The Spearman correlation test was used to determine the correlation between two variables containing continuous data. Statistical significance was set at $p$ less than 0.05 . 


\section{Results}

Sociodemographic, clinical, radiological, and ultrasonographic characteristics of the studied patients are illustrated in Tables 1 and 2. The mean age of the studied patients was $49.65 \pm 6.84$ years, and the majority of them were females $(82.5 \%)$. According to KL grading, the largest percent of patients had grade II KOA (45\%) followed by grade III (40\%). Moreover, ultrasonographic GFC grading showed that $40 \%$ had grade $2 \mathrm{~b}$, followed by grade $2 \mathrm{a}$ in $30 \%$ of patients.

Correlations between clinical characteristics and either radiological or ultrasonographic findings of the studied patients are presented in Tables 3. The KL grading had significant positive correlations with age and disease duration as well as WOMAC scores $(p=<0.001,<0.001$ and $<0.001$, respectively). Moreover, the GFC grading had significant

Table 1 Demographic and clinical characteristics of the studied patients

\begin{tabular}{|c|c|}
\hline Data characteristics & \\
\hline \multicolumn{2}{|l|}{ Gender } \\
\hline Male, $n(\%)$ & $7(17.5)$ \\
\hline Female, $n(\%)$ & $33(82.5)$ \\
\hline Age (years) (mean $\pm S D)$ & $49.65 \pm 6.84$ \\
\hline Disease duration (years) (mean $\pm S D$ ) & $6.60 \pm 4.18$ \\
\hline Height $(\mathrm{cm})$ & $163.17 \pm 6.84$ \\
\hline Weight (kg) & $87.05 \pm 11.66$ \\
\hline BMI $\left(\mathrm{kg} / \mathrm{m}^{2}\right)($ mean $\pm S D)$ & $32.74 \pm 3.88$ \\
\hline \multicolumn{2}{|l|}{ Tenderness, $n(\%)$} \\
\hline No & $2(5)$ \\
\hline Yes & $38(95)$ \\
\hline \multicolumn{2}{|l|}{ Alignment, $n(\%)$} \\
\hline Normal & $21(52.5)$ \\
\hline Varus & $18(45)$ \\
\hline Valgus & $1(2.5)$ \\
\hline \multicolumn{2}{|l|}{ Quadriceps muscle wasting, $n(\%)$} \\
\hline Yes & $16(40)$ \\
\hline No & $24(60)$ \\
\hline \multicolumn{2}{|l|}{ Range of motion, $n(\%)$} \\
\hline Full & $27(67.5)$ \\
\hline Limited flexion & $12(30)$ \\
\hline Limited extension & $1(2.5)$ \\
\hline \multicolumn{2}{|l|}{ Stability, $n(\%)$} \\
\hline Stable & $27(67.5)$ \\
\hline Lax & $13(32.5)$ \\
\hline WOMAC pain subscale (mean \pm SD) & $8.80 \pm 3.107$ \\
\hline WOMAC stiffness subscale (mean \pm SD) & $2.00 \pm 1.664$ \\
\hline WOMAC function subscale (mean \pm SD) & $28.13 \pm 11.062$ \\
\hline WOMAC total score (mean $\pm S D)$ & $38.83 \pm 14.74$ \\
\hline
\end{tabular}

Table 2 Radiographic and US characteristics of the studied patients

\begin{tabular}{|c|c|}
\hline \multicolumn{2}{|c|}{ Radiographic (KL) grade, $n$ (\%) } \\
\hline 1 & $3(7.5)$ \\
\hline$\|$ & $18(45)$ \\
\hline III & $16(40)$ \\
\hline IV & $3(7.5)$ \\
\hline \multicolumn{2}{|c|}{ Ultrasonographic features, $n(\%)$} \\
\hline \multicolumn{2}{|c|}{ GFC grade } \\
\hline 1 & $7(17.5)$ \\
\hline $2 a$ & $12(30)$ \\
\hline $2 b$ & $16(40)$ \\
\hline 3 & $5(12.5)$ \\
\hline \multicolumn{2}{|c|}{ Lateral osteophyte grading } \\
\hline 1 & $13(32.5)$ \\
\hline 2 & $23(57.5)$ \\
\hline 3 & $4(10)$ \\
\hline \multicolumn{2}{|c|}{ Medial osteophyte grading } \\
\hline 0 & $2(5)$ \\
\hline 1 & $10(25)$ \\
\hline 2 & $14(35)$ \\
\hline 3 & $14(35)$ \\
\hline \multicolumn{2}{|l|}{ MME } \\
\hline$<0.3$ & $18(45)$ \\
\hline$\geq 0.3$ & $22(55)$ \\
\hline \multicolumn{2}{|l|}{ LME } \\
\hline$<3 \mathrm{~mm}$ & $28(70)$ \\
\hline$\geq 3 \mathrm{~mm}$ & $12(30)$ \\
\hline \multicolumn{2}{|l|}{ Effusion } \\
\hline Yes & $34(85)$ \\
\hline No & $6(15)$ \\
\hline \multicolumn{2}{|c|}{ Baker's cyst } \\
\hline Yes & $11(27.5)$ \\
\hline No & $29(72.5)$ \\
\hline
\end{tabular}

$S D$ standard deviation, GFC global femoral cartilage, MME medial meniscal extrusion, LME lateral meniscal extrusion

positive correlation with age, disease duration, and all WOMAC subscales (pain, stiffness, functional, and total $p=<0.001,<0.001,0.040,<0.001,<0.001$, and $<0.001)$. Significant positive correlations were found between other US parameters and all WOMAC subscales (pain, stiffness, functional, and total) except LME which was significantly correlated with WOMAC stiffness subscale only $(p=0.018)$.

Correlations between radiographic and ultrasonographic characteristics of KOA in the studied patients are illustrated in Table 4 and Figs. 1 and 2. There were significant positive correlations between KL grading and all US parameters. 
Table 3 Correlation between clinical, radiological, and ultrasonographic parameters

\begin{tabular}{lllllll}
\hline & K-L & GFC & $\begin{array}{l}\text { MME } \\
\boldsymbol{r}(\boldsymbol{P})\end{array}$ & $\begin{array}{l}\text { LME } \\
\boldsymbol{r}(\boldsymbol{P})\end{array}$ & $\begin{array}{l}\text { Med. osteophyte } \\
\boldsymbol{r}(\boldsymbol{P})\end{array}$ & $\begin{array}{l}\text { Lat. osteophyte } \\
\boldsymbol{r}(\boldsymbol{P})\end{array}$ \\
\hline WOMAC pain & $0.456\left(0.003^{*}\right)$ & $0.327\left(0.040^{*}\right)$ & $0.386\left(0.014^{*}\right)$ & $0.287(0.073)$ & $0.352\left(0.026^{*}\right)$ & $0.441\left(0.004^{*}\right)$ \\
WOMAC stiffness & $0.641\left(<0.001^{*}\right)$ & $0.558\left(<0.001^{*}\right)$ & $0.390\left(0.013^{*}\right)$ & $0.374\left(0.018^{*}\right)$ & $0.457\left(0.003^{*}\right)$ & $0.452\left(0.003^{*}\right)$ \\
WOMAC function & $0.732\left(<0.001^{*}\right)$ & $0.615\left(<0.001^{*}\right)$ & $0.430\left(0.006^{*}\right)$ & $0.172(0.290)$ & $0.562\left(<0.001^{*}\right)$ & $0.568\left(<0.001^{*}\right)$ \\
WOMAC total score & $0.736\left(<0.001^{*}\right)$ & $0.607\left(<0.001^{*}\right)$ & $0.413\left(0.008^{*}\right)$ & $0.202(0.211)$ & $0.541\left(<0.001^{*}\right)$ & $0.573\left(<0.001^{*}\right)$ \\
\hline
\end{tabular}

WOMAC score Western Ontario and McMaster Universities Osteoarthritis score, GFC global femoral cartilage, MME medial meniscal extrusion, $L M E$ lateral meniscal extrusion, $r$ Spearman's correlation, $p p$ value for comparing between the studied groups

*Statistically significant at $p \leq 0.05$

Table 4 Correlation between radiological and ultrasound parameters

\begin{tabular}{lll}
\hline Ultrasound features & KL grading score & \\
& $\boldsymbol{R}$ & $(\boldsymbol{p})$ \\
\hline GFC & 0.794 & $<0.001^{*}$ \\
MME & 0.504 & $0.001^{*}$ \\
LME & 0.320 & $0.044^{*}$ \\
Med. osteophyte grading & 0.632 & $<0.001^{*}$ \\
Lat. osteophyte grading & 0.614 & $<0.001^{*}$ \\
\hline
\end{tabular}

GFC global femoral cartilage, MME medial meniscal extrusion, $L M E$ lateral meniscal extrusion. $p p$ value for comparing between the studied groups

*Statistically significant at $p \leq 0.05$

\section{Discussion}

Cartilage breakdown, subchondral bone alterations, and formation of osteophytes are the key points in the natural development (cascade) of $\mathrm{KOA}$, with pain and loss of function counting as major devastating consequences [23]. Hence, sensitive tools for their development and progression are mandatory. Conventional radiography has long been the major tool for assessment of KOA; however, it is an imprecise guide to the likelihood that knee pain or disability will be present especially in the early and mild cases [24]. Several studies have argued against the association between radiographic grading of $\mathrm{KOA}$ and pain severity and function as assessed by WOMAC. Other studies [25-27] found no correlation between radiographic grading and neither functional status using Modified Lower Extremity Functional Scale nor disability as assessed by the Health Assessment Questionnaire (HAQ) or WOMAC score. Even the radiographic osteophyte length was not correlated with pain severity in another study by Creamer et al. [28]. On the other hand, Muraki et al. [29] found a strong association between knee pain and radiological joint space narrowing. These inconsistent results across studies elucidate that conventional radiography may fail to detect structural changes associated with pain in early KOA, especially with patients of mild to moderate severity. It does
Table 5 Multivariate linear regression analysis for the ultrasonographic parameters affecting WOMAC total score and WOMAC subscores

\begin{tabular}{|c|c|c|c|c|}
\hline & \multicolumn{2}{|c|}{ Multivariate } & \multirow[b]{2}{*}{$S E$} & \multirow[b]{2}{*}{$T$} \\
\hline & Sig. & $B$ & & \\
\hline \multicolumn{5}{|l|}{ WOMAC total score } \\
\hline GFC & 0.004 & 7.591 & 2.489 & 3.049 \\
\hline Med. osteophyte & 0.087 & 4.356 & 2.477 & 1.758 \\
\hline LME & 0.928 & -1.163 & 12.719 & -0.091 \\
\hline \multicolumn{5}{|l|}{$R^{2}=0.445$} \\
\hline \multicolumn{5}{|l|}{$F=9.628, p=<0.001^{*}$} \\
\hline \multicolumn{5}{|l|}{ WOMAC stiffness score } \\
\hline GFC & 0.020 & 0.730 & 0.300 & 2.431 \\
\hline Med. osteophyte & 0.402 & 0.254 & 0.299 & 0.849 \\
\hline LME & 0.164 & 2.182 & 1.535 & 1.422 \\
\hline \multicolumn{5}{|l|}{$R^{2}=0.366$} \\
\hline \multicolumn{5}{|l|}{$F=6.932, p=0.001^{*}$} \\
\hline \multicolumn{5}{|c|}{ WOMAC physical function score } \\
\hline GFC & $0.003^{*}$ & 5.978 & 1.865 & 3.206 \\
\hline Med. osteophyte & 3.294 & 0.084 & 1.855 & 1.776 \\
\hline LME & -6.529 & 0.498 & 9.527 & -0.685 \\
\hline \multicolumn{5}{|l|}{$R^{2}=0.447$} \\
\hline \multicolumn{5}{|c|}{$F=9.704, p=<0.001^{*}$} \\
\hline \multicolumn{5}{|l|}{ WOMAC pain score } \\
\hline GFC & 0.149 & 0.885 & 0.885 & 1.473 \\
\hline Med. osteophyte & 0.187 & 0.805 & 0.805 & 1.346 \\
\hline LME & 0.301 & 3.219 & 3.219 & 1.049 \\
\hline \multicolumn{5}{|l|}{$R^{2}=0.272$} \\
\hline$F=4.494, p=0.009^{*}$ & & & & \\
\hline
\end{tabular}

*Statistically significant at $p \leq 0.05$

WOMAC score Western Ontario and McMaster Universities Osteoarthritis score, GFC global femoral cartilage, MME medial meniscal extrusion, $L M E$ lateral meniscal extrusion

not adequately depict articular cartilage and other soft tissue damage that patients mostly categorize [30].

Since then more research has been conducted on tools more accurately depicting early cartilage and soft tissue damage as MRI and US. Since MRI is an 


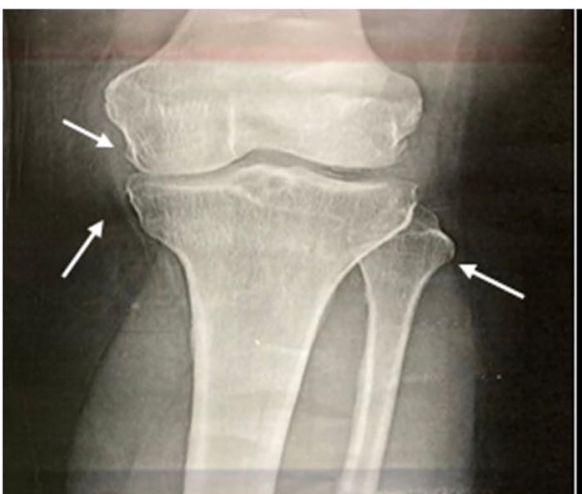

(a)

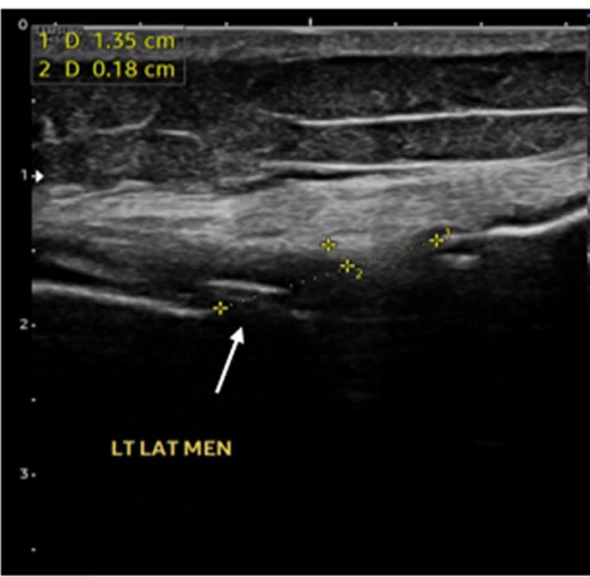

(c)

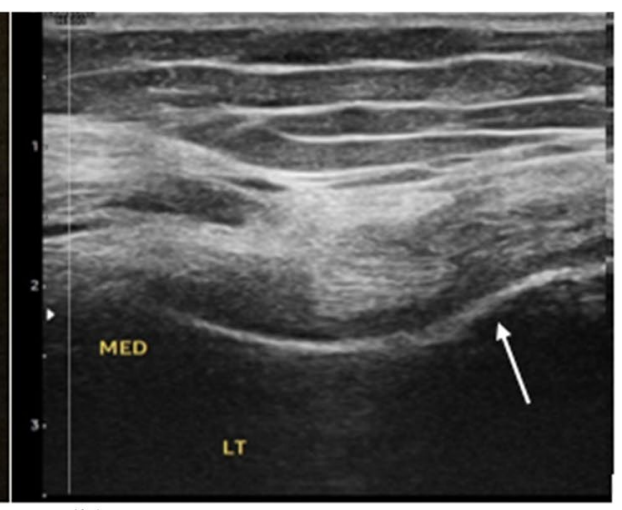

(b)

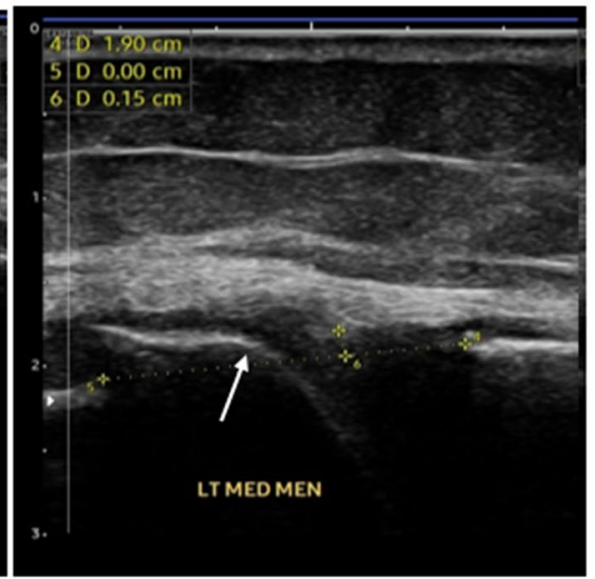

(d)

Fig. 1 a Plain X-ray of left knee joint anteroposterior 48 years old female patient showing grade $2 \mathrm{KL}$ grading with presence of definite osteophytes and possible joint space narrowing. Sonographic assessment showed $\mathbf{b}$ global femoral cartilage (GFC) semi-quantitative grading G2b with loss of normal cartilage interfaces sharpness and increased echogenecity of the cartilage together with local thinning ( $>50 \%$ but $<100 \%$ of the catilage), $\mathbf{c}$ lateral medium osteophyte grade 2 and lateral menicial extrusion measuring $1.8 \mathrm{~mm}$, and $\mathbf{d}$ medial large osteophyte grade 3 and medial meniscial extrusion measuring $1.5 \mathrm{~mm}$

expensive tool, and not readily available in any clinical setting, more effort was invested with US and its capability in defining those early soft tissue changes in KOA especially the ones related to pain and eventually leading to loss of function as concluded by Abicalaf et al. [31]. Recently ultrasound has become popular among rheumatologists as the first-choice imaging for the evaluation and monitoring of KOA.

To evaluate the ability of US in assessing severity of KOA, we measured US GFC grade and lateral and medial osteophyte grade as well as medial and lateral meniscal extrusion together with radiographic KL grading. Significant correlation was found between radiographic KL grading and pain and function as assessed by WOMAC score, as well as between radiographic KL grading and all US findings which was of moderate strength with all sonographic parameters, except for GFC which was of high strength $(r>0.75)$. Also, a study of Mortada et al.
[32] found good agreement between KL grading scale and a proposed simple and reliable US grading scale based on medial osteophytic grading. Interestingly, Malas et al. in 2014 [33] reported that a positive correlation was discovered between US meniscal extrusion measurements and radiological KL grade. This was also reported by Brom et al. [34] concluding that US is a valid method with high sensitivity and specificity for detecting radiographic KOA.

As for correlation between US parameters and WOMAC scores of pain and function, there was also significant correlation with all US findings including GFC, MME, and medial and lateral osteophyte grading except LME which was only correlated with WOMAC stiffness subscale. To detect the most independent ultrasonographic variables predictors for WOMAC grading severity, multivariate linear regression analysis was performed. Cartilage damage graded by GFC was the only 


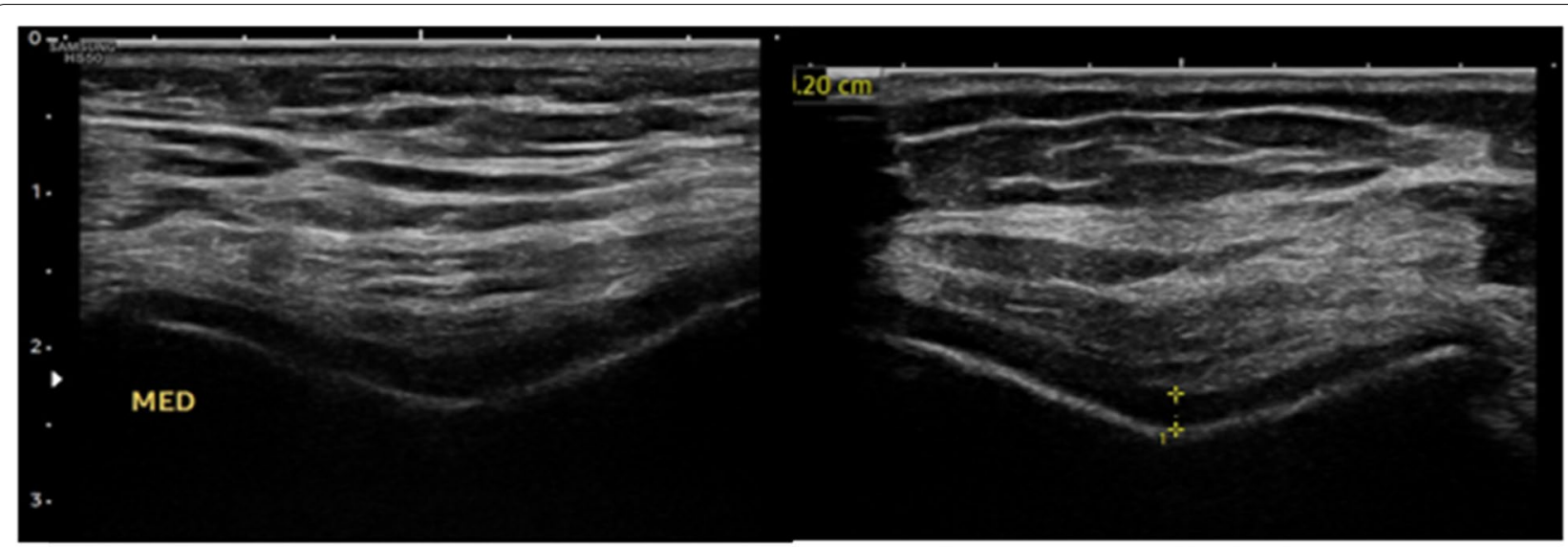

(a)

(b)
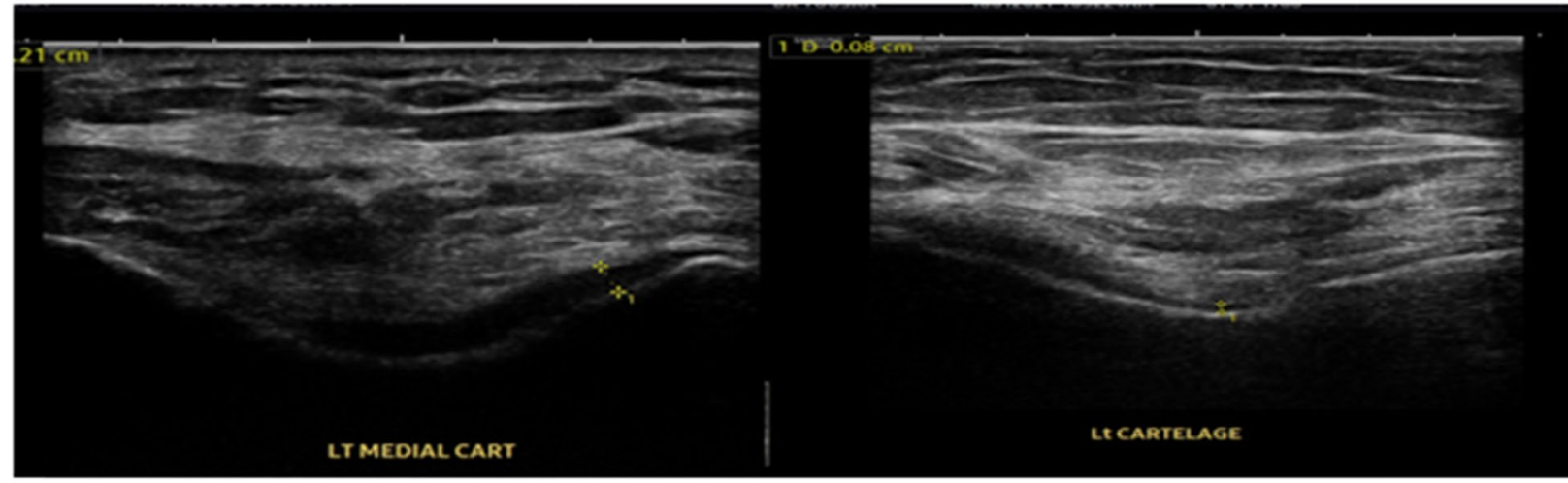

(c)

(d)

Fig. 2 Transversal scan of the femoral condyle for assessing the global femoral cartilage: a grade 1, b grade $2 A, \mathbf{c}$ grade $2 B$, and $\mathbf{d}$ grade 3 . Multivariate linear stepwise regression analysis was performed to detect the most independent ultrasonographic variable predictor for WOMAC grading severity (Table 5, Fig. 3). Cartilage damage graded by GFC was the only independent predictor for WOMAC total score and WOMAC subscales; stiffness and physical function are relative to other ultrasonographic variables. WOMAC pain score was predicted only with the lateral osteophyte

independent predictor for WOMAC total score and WOMAC subscales; stiffness and physical function are relative to other ultrasonographic variables. On the other hand, lateral osteophyte grading was the only predictor of WOMAC pain score. This finding is supported by the fact that hyaline cartilage has not got pain fibers and so cannot be the source of pain [28], while growing osteophytes show neovascularization, which can be accompanied by sensory nerve growth and therefore be connected to pain generation [35]. In addition to this, osteophyte stimulation of sensory nerve terminals in the neighboring synovium can also induce bone-related pain [36]. In a study by Serban et al. [27], they reported that cartilage damage score was an independent predictor for both VAS and WOMAC scores. Medial osteophytes were associated with pain at palpation and are independent predictors for the WOMAC score. Lateral meniscal protrusion was also a predictor for the WOMAC score. Interestingly, Mortada et al. reported that the medial femoral osteophyte, which represents the degenerative domain of the novel proposed ultrasonographic ZAGAZIG scale, was well correlated with WOMAC subscales (pain, stiffness and function) [37]. Also, Chen et al. [38] found that the cartilage damage on US was linked to worse WOMAC and Lequesne index scores. Also, Kijima et al. [39] found that the amount of MME was correlated to pain intensity. Our results was also supported by Podlipská et al. in 2017 [21] who reported that early cartilage changes seen in US lead to pain in KOA, but in contrast to our results, they found no significant association with meniscal extrusion suggesting that the extruded meniscus was simply a coexisting OA feature rather than painful and disabling pathological processes within the knee in symptomatic patients. Similarly, Razek et al. in 2016 [40] reported that 


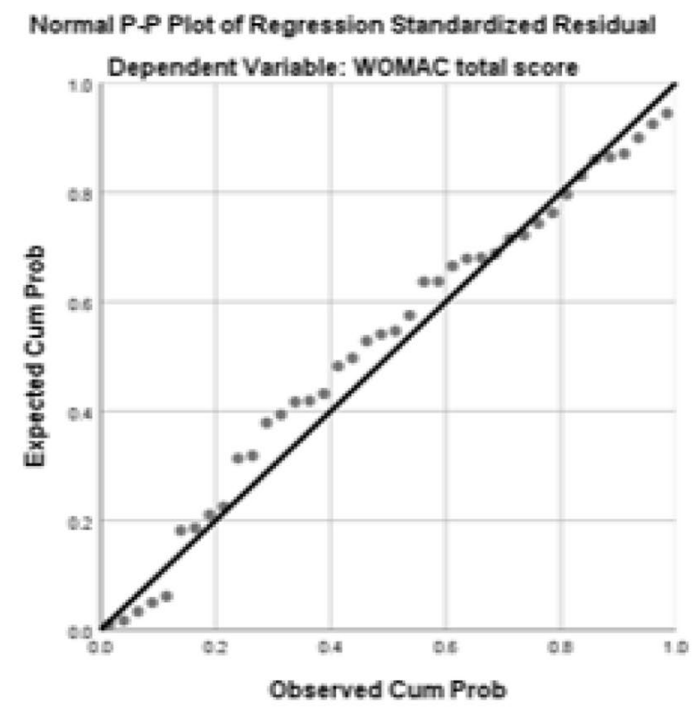

Normal P-P Plot of Regression Standardized Residual Dependent Variable: WOMAC Physical Function subscale

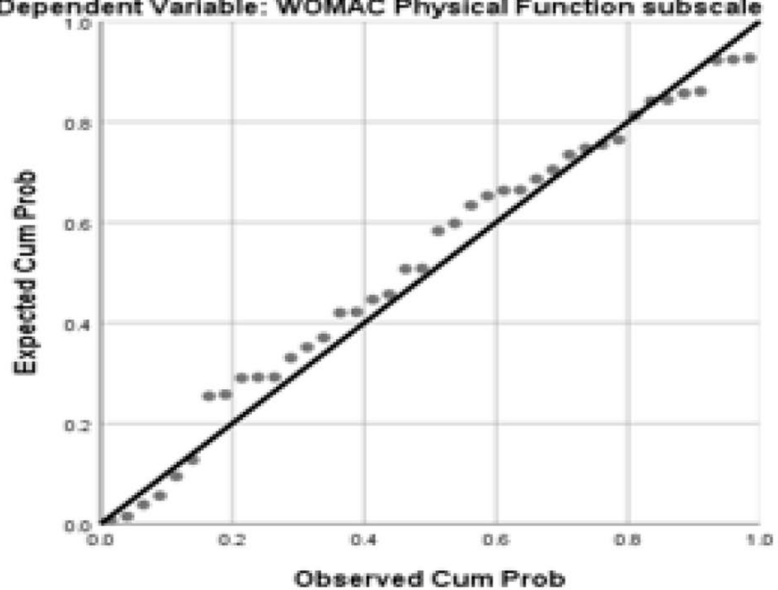

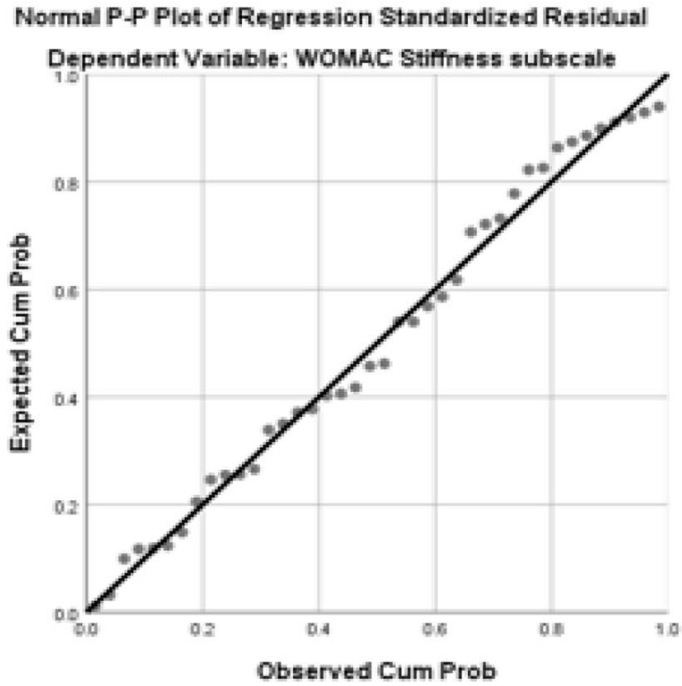

Normal P.P Plot of Regression Standardized Residual

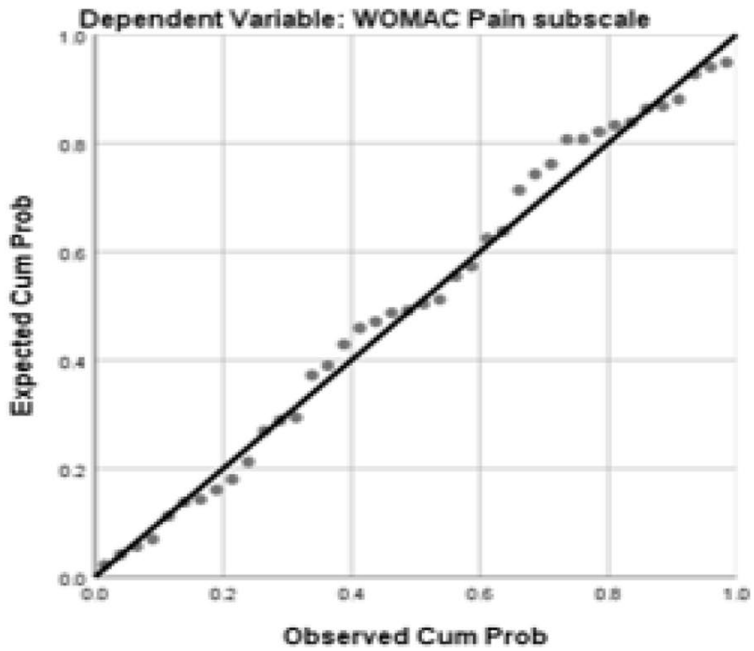

Fig. 3 Multivariate linear regression analysis for the parameters affecting WOMAC total score and WOMAC subscales

US characteristics of cartilage damage, osteophytes, and synovial effusion were related to poorer WOMAC scores. On the contrary, Malas et al. [33] did not find a positive association between WOMAC scales and the severity of cartilage damage.

This study has some limitations. Firstly, with a small sample size, more researches on a large number of patients are needed to determine the validity of this study. Second, the lack of data on the patients' treatment is one of the study's significant limitations: chronic use of antiinflammatories or analgesics can alter pain perception. Third, we did not compare our findings to those of a control group (asymptomatic with radiographic OA). Fourth, ultrasonography assessment measures were conducted only once by one reader. Fifth is the lack of comparison of US findings with MRI findings, as we did not regard the presence of bone marrow lesions as a significant source of pain in KOA. The link between KOA symptoms and US findings is still unclear and contentious, and it has to be investigated further in larger research.

\section{Conclusions}

GFC was the only independent predictor for WOMAC loss of function, and lateral osteophyte grading was the only predictor for WOMAC pain score. US can visualize multiple aspects of the joint and was significantly 
correlated in this study with radiographic assessment, and by time, radiography may not be the method of choice.

\author{
Abbreviations \\ OA: Osteoarthritis; KOA: Knee osteoarthritis; K-L: Kellgren-Lawrence (K-L) \\ scale; US: Ultrasound; GFC: Global femoral hyaline cartilage; WOMAC: Western \\ Ontario and McMaster Universities Osteoarthritis Index scale; CR: Conventional \\ radiography; ICF: International Classification of Functioning, Disability and \\ Health; MME: Medial meniscal extrusion; LME: Lateral meniscal extrusion; VAS: \\ Visual analog scale.
}

\section{Acknowledgements}

The authors gratefully acknowledge Yousra Hisham Abdel-Fattah for sharing in the ultrasonographic assessments.

\section{Authors' contributions}

ES S performed the sonographic assessment and contributed to the interpretation of data and work edit. AM M put the idea, design of the work, and general supervision on the whole work. E R performed the interpretation of data and work edit. TR performed the routine investigations assessment and participated in revising and editing the work. WF N contributed to the clinical assessment, interpretation of data, and writing of the manuscript. All authors read and approved the final manuscript.

\section{Funding}

The authors received no specific funding for this work.

\section{Availability of data and materials}

The datasets used and/or analyzed during the current study are available from the corresponding author on reasonable request.

\section{Declarations}

\section{Ethics approval and consent to participate}

The local Ethics Committee of Faculty of Medicine, Alexandria University, Egypt (IRB NO: 00012098-FWA NO: 00018699), approved the study. Date of approval: 16 July 2020. Serial number: 0201371. Informed consent was obtained from every patient.

\section{Consent for publication}

Not applicable

\section{Competing interests}

The authors declare that they have no competing interests.

\section{Author details}

'Physical Medicine, Rheumatology and Rehabilitation Department, Faculty of Medicine, Alexandria University, Alexandria, Alexandria Governorate 21131, Egypt. ${ }^{2}$ Clinical and Chemical Pathology Department, Faculty of Medicine, Alexandria University, Alexandria, Alexandria Governorate 21131, Egypt.

Received: 31 December 2021 Accepted: 9 February 2022

Published online: 03 March 2022

\section{References}

1. Lawrence RCFD, Helmick CG et al (2008) Estimates of the prevalence of arthritis and other rheumatic conditions in the United States. Part II. Arthritis Rheum 58(1):26-35

2. Arden N, Nevitt MC (2006) Osteoarthritis: epidemiology. Best Pract Res Clin Rheumatol 20(1):3-25

3. Salaffi F, Ciapetti A, Carotti M (2014) The sources of pain in osteoarthritis: a pathophysiological review. Reumatismo 66(1):57-71

4. Bedson J, Croft PR (2008) The discordance between clinical and radiographic knee osteoarthritis: a systematic search and summary of the literature. BMC Musculoskelet Disord 9(1):1-11
5. Bevers K, Bijlsma JW, Vriezekolk JE, van den Ende CH, den Broeder AA (2014) Ultrasonographic features in symptomatic osteoarthritis of the knee and relation with pain. Rheumatology 53(9):1625-1629

6. Spector T, Hart D, Byrne J, Harris P, Dacre J, Doyle D (1993) Definition of osteoarthritis of the knee for epidemiological studies. Ann Rheumatic Dis 52(11):790-794

7. Dougados M, Gueguen A, Nguyen M, Thiesce A, Listrat V, Jacob L et al (1992) Longitudinal radiologic evaluation of osteoarthritis of the knee. J Rheumatol 19(3):378-384

8. Kellgren JH, Lawrence J (1957) Radiological assessment of osteo-arthrosis. Ann Rheum Dis 16(4):494

9. Braun HJ, Gold GE (2012) Diagnosis of osteoarthritis: imaging. Bone 51(2):278-288

10. Seifeldein GS, Haseib A, Hassan HA, Ahmed G (2019) Correlation of knee ultrasonography and Western Ontario and McMaster University (WOMAC) osteoarthritis index in primary knee osteoarthritis. Egypt J Radiol Nucl Med 50(1):1-8

11. Wakefield R, Gibbon W, Emery P (1999) The current status of ultrasonography in rheumatology. Rheumatology (Oxford, England) 38(3):195-198

12. Nelson AE (2020) Turning the page in osteoarthritis assessment with the use of ultrasound. Curr Rheumatol Rep 22(10):1-8

13. Altman R (1991) Criteria for classification of clinical osteoarthritis. J Rheumatol Suppl 27:10-12

14. Rauch A, Cieza A, Stucki G (2008) How to apply the International Classification of Functioning, Disability and Health (ICF) for rehabilitation management in clinical practice. Eur J Phys Rehabil Med 44(3):329-342

15. Doyle D, Dieppe P, Scott J, Huskisson E (1981) An articular index for the assessment of osteoarthritis. Ann Rheum Dis 40(1):75-78

16. Lequesne M (1991) Indices of severity and disease activity for osteoarthritis. In Sem Arthritis Rheumatism 20(6):48-54.

17. Escobar A, Quintana J, Bilbao A, Arostegui I, Lafuente I, Vidaurreta I (2007) Responsiveness and clinically important differences for the WOMAC and SF-36 after total knee replacement. Osteoarthritis Cartilage 15(3):273-280

18. Guermazi M, Poiraudeau S, Yahia M, Mezganni M, Fermanian J, Elleuch MH et al (2004) Translation, adaptation and validation of the Western Ontario and McMaster Universities osteoarthritis index (WOMAC) for an Arab population: the Sfax modified WOMAC. Osteoarthritis Cartilage 12(6):459-468

19. JK K, Lawrence JS (1957) Radiological assessment of osteoarthritis. Ann Rheum Dis 16:494-501

20. Koski JM, Kamel A, Waris P, Waris V, Tarkiainen I, Karvanen E et al (2016) Atlas-based knee osteophyte assessment with ultrasonography and radiography: relationship to arthroscopic degeneration of articular cartilage. Scand J Rheumatol 45(2):158-164

21. Podlipská J, Koski JM, Kaukinen P, Haapea M, Tervonen O, Arokoski JP et al (2017) Structure-symptom relationship with wide-area ultrasound scanning of knee osteoarthritis. Sci Rep 7(1):1-10

22. IBM SPSS Statistics for Windows VA, NY (2011): IBM Corp.

23. Jiang Y, Zhu L, Zhang T, Lu H, Wang C, Xue B et al (2017) BRD4 has dual effects on the HMGB1 and NF-KB signalling pathways and is a potential therapeutic target for osteoarthritis. Biochim Biophys Acta Mole Basis Dis 1863(12):3001-3015

24. Heidari B (2011) Knee osteoarthritis prevalence, risk factors, pathogenesis and features: Part I. Caspian J Int Med 2(2):205

25. Ay S, Evcik D (2008) Effectiveness of pain, disease severity and radiological grading on disability of daily living activities in knee osteoarthritis.

26. Barker K, Lamb SE, Toye F, Jackson S, Barrington S (2004) Association between radiographic joint space narrowing, function, pain and muscle power in severe osteoarthritis of the knee. Clin Rehabil 18(7):793-800

27. Serban O, Porojan M, Deac M, Cozma F, Solomon C, Lehghel M et al (2016) Pain in bilateral knee osteoarthritis-correlations between clinical examination, radiological, and ultrasonographical findings. Med Ultrasonography 18(3):318-325

28. Creamer P, Lethbridge-Cejku M, Hochberg M (2000) Factors associated with functional impairment in symptomatic knee osteoarthritis. Rheumatology 39(5):490-496

29. Muraki S, Oka H, Akune T, Mabuchi A, En-Yo Y, Yoshida M et al (2009) Prevalence of radiographic knee osteoarthritis and its association with knee pain in the elderly of Japanese population-based cohorts: the ROAD study. Osteoarthritis Cartilage 17(9):1137-1143 
30. Hodler J, Resnick D (1996) Current status of imaging of articular cartilage. Skeletal Radiol 25(8):703-709

31. Abicalaf CARP, Nakada LN, Dos Santos FRA, Akiho I, Dos Santos ACA, Imamura M et al (2021) Ultrasonography findings in knee osteoarthritis: a prospective observational cross-sectional study of 100 patients. Sci Rep 11(1):1-9

32. Mortada M, Zeid A, Al-Toukhy MAE-h, Ezzeldin N, Elgawish M. (2016) Reliability of a proposed ultrasonographic grading scale for severity of primary knee osteoarthritis. Clin Med Insights Arthritis Musculoskel Disord 9:S38141

33. Malas FÜ, Kara M, Kaymak B, Akıncı A, Özçakar L (2014) Ultrasonographic evaluation in symptomatic knee osteoarthritis: clinical and radiological correlation. Int J Rheum Dis 17(5):536-540

34. Brom M, Gandino IJ, Zacariaz Hereter JB, Scolnik M, Mollerach FB, Ferreyra Garrott LG et al (2020) Performance of ultrasonography compared to conventional radiography for the diagnosis of osteoarthritis in patients with knee pain. Front Med 7:319

35. Mapp PI, Walsh DA (2012) Mechanisms and targets of angiogenesis and nerve growth in osteoarthritis. Nat Rev Rheumatol 8(7):390-398

36. Hunter DJ, McDougall JJ, Keefe FJ (2008) The symptoms of osteoarthritis and the genesis of pain. Rheum Dis Clin North Am 34(3):623-643

37. Mortada M, Dawa GA, Amer YA (2021) Construct validity of a clinically correlated knee osteoarthritis ultrasonographic scale: a cross-sectional observational study. BMJ open 11(12):e048444

38. Chen Y-J, Chen C-H, Wang C-L, Huang M-H, Chen T-W, Lee C-L (2015) Association between the severity of femoral condylar cartilage erosion related to knee osteoarthritis by ultrasonographic evaluation and the clinical symptoms and functions. Arch Phys Med Rehabil 96(5):837-844

39. Kijima H, Yamada S, Nozaka K, Saito H, Shimada Y (2015) Relationship between pain and medial meniscal extrusion in knee osteoarthritis. Adv Orthop 2015:210972

40. Razek AAKA, El-Basyouni SR (2016) Ultrasound of knee osteoarthritis: interobserver agreement and correlation with Western Ontario and McMaster Universities Osteoarthritis. Clin Rheumatol 35(4):997-1001

\section{Publisher's Note}

Springer Nature remains neutral with regard to jurisdictional claims in published maps and institutional affiliations.

\section{Submit your manuscript to a SpringerOpen ${ }^{\circ}$ journal and benefit from:}

- Convenient online submission

- Rigorous peer review

- Open access: articles freely available online

- High visibility within the field

- Retaining the copyright to your article 